\title{
Theoretical Analysis of the Nutritional Value of Beet Biscuits (Beta Vulgaris) and Their Consumption and Marketing Trends
}

\section{Análisis Teórico del Valor Nutritivo en las Galletas de Remolacha (Beta Vulgaris) y Su Tendencia de Consumo y Comercialización}

I International Seminar of Livestock and Agroindustrial Production ESPOCH 2020

Corresponding Author:

B. Bravo

maria.bravo@espoch.edu.ec

Published: 2 September 2021

Production and Hosting by

Knowledge E

(c) B. Bravo and M.

Miranda. This article is

distributed under the terms of the Creative Commons

Attribution License, which permits unrestricted use and redistribution provided that the original author and source are credited.

\section{B. Bravo and M. Miranda}

Escuela de Ingeniería en Industrias Pecuarias. Facultad de Ciencias Pecuarias, Escuela Superior Politécnica de Chimborazo, Riobamba, Ecuador

\section{Abstract}

This study aimed to examine the nutritional value of beet biscuits and the trends in their consumption and marketing. Currently, adults, adolescents and children consume this vegetable without the intervention of any industrial process. To determine the nutritional value of beet biscuits, information from existing research was sought for a detailed comparison of the beet cookie and the stuffed and festival cookies. Based on these analyses, it was concluded that beet cookies have a considerable nutritional value, which provides important health benefits to the people who consume them. Nowadays, the desire to maintain a healthy diet is a trend, due to the risk of illnesses derived from inappropriate eating habits. The biscuit industry was analyzed, showing a growing trend in the marketing of biscuits. The main competitive advantage of beets is the vitamins they offer.

Keywords: nutrition, health, consumption, marketing.

\section{Resumen}

La presente investigación tiene como objetivo realizar un análisis del valor nutritivo en galletas de remolacha, la tendencia de consumo y comercialización. En la actualidad adultos, adolescentes y niños consumen esta hortaliza, en forma natural, sin la intervención de ningún proceso industrial. Para determinar su valor nutricional se buscó información de investigaciones existentes para un análisis minucioso entre la galleta de remolacha y otras galletas de similares características; esta investigación es de tipo analítica y comparativa, el análisis bibliográfico realizado con información de fuentes secundarias y la comparación nutricional entre ellas permitió definir, que las galletas de remolacha tienen una riqueza nutricional muy considerable, que aporta importantes beneficios a la salud de las personas que las consumen. Actualmente es tendencia el deseo de mantener una alimentación saludable, mediante la elección de productos sanos, debido a las enfermedades derivadas por hábitos alimentarios inadecuados. Se analizó la industria de la galletería, presentando una predisposición creciente en la comercialización de galletas, por lo que se prioriza las vitaminas que ofrecen los vegetales en esta investigación específica con la remolacha se destaca como principal ventaja competitiva.

Palabras Clave: nutrición, salud, consumo, comercialización. 


\section{Introducción}

Tomando en cuenta que en los últimos años se ha demostrado el consumo excesivo de azúcares sea en bebidas, chocolates y entre ellos: galletas de sal y dulce industrializados, con un alto contenido de carbohidratos, ha desembocado en distintas enfermedades, como la diabetes, producida por una mala alimentación, principalmente por la ingesta de productos con alto contenido de azúcar la misma, que afecta a adultos mayores, niños y adolescentes quienes están propensos a este tipo de enfermedad [1].

Después de varias investigaciones, se estima que su prevalencia es de 150 millones de personas, lo cual constituye aproximadamente el 2,5\% de la población mundial [2]. Además, el consumo de gluten que se encuentra en el trigo, que forma parte de la elaboración de las galletas, provocan ciertas enfermedades como el estreñimiento, pues interfiere en el metabolismo y la absorción de nutrientes de los alimentos.

El objetivo es ofrecer galletas saludables, elaboradas a base de vegetales, como la remolacha, la cual aporta vitaminas y nutrientes, mediante un estudio comparativo nutricional, así como también la tendencia de consumo y su comercialización.

\subsection{Antecedentes de la remolacha}

La primera referencia histórica sobre la remolacha proviene de Babilonia en el siglo VIII AC., y la primera descripción proviene de Aristóteles (350 años AC.) aunque hay referencias del Antiguo Egipto, donde usaban las momias quemadas para conseguir un fino carbón, que, molido, se utilizaba para refinar y blanquear azúcar [3].

Es muy conocido que la remolacha es rica en azúcar, en 1747 se demostró que los cristales de sabor dulce obtenidos del jugo de la remolacha eran iguales a los de la caña de azúcar [4]. Es una planta perene que produce hojas y raíces para el consumo humano o animal.

En la remolacha roja se encuentra la betanina que corresponde a un 75-95\% de los pigmentos, los otros son isobetanina, prebetanina, los dos últimos son monoesteres sulfatadas de la betanina e isobetanina, respectivamente. Los pigmentos amarillos más abundantes en la remolacha son vulgaxantina I y II. La presencia de betalainas en plantas es mutuamente excluyente de la de antocianinas [5].

\subsection{Propiedades nutricionales}

La remolacha tiene propiedades anti anémico también podría reducir la presión arterial. La remolacha es una fuente de vitamina $\mathrm{A}$ y $\mathrm{C}$, hierro y otros minerales, carotenoides y fibra dietética. La remolacha también presenta pigmentos naturales causante del color rojo, hay que destacar también que la remolacha es fundamental en la dieta de los vegetarianos y de los veganos, por ser una excelente fuente de hierro y ácido fólico [6]. 
Según una investigación realizada [7]:

[...] la remolacha es particularmente ricas en folato. Se ha encontrado que el ácido fólico proviene defectos de nacimiento del tubo neuronal y ayuda contra enfermedades cardiacas y anemia. La remolacha también tiene alto contenido de fibra, soluble einsoluble. La fibra insoluble ayuda a mantener su tracto intestinal trabajando perfectamente, mientras que la fibra soluble mantiene sus niveles de azúcar en la sangre y colesterol controlados. La remolacha es un alimento de moderado contenido calórico, ya que, tras el agua, los hidratos de carbono son el componente más abundante, lo que hace que ésta sea una de las hortalizas más ricas en azúcares.

Las remolachas contienen una gran cantidad de nitratos inorgánicos. Estos compuestos son el precursor de una molécula de señalización muy importante que nuestro cuerpo necesita para funcionar (óxido nítrico). El óxido nítrico no se produce de forma natural en nuestro organismo, pero podemos aumentar drásticamente su disponibilidad por el consumo de alimentos ricos en nitrato. Entre otras funciones, el óxido nítrico actúa como vasodilatador en el funcionamiento del músculo esquelético, y aumentando el tamaño de los vasos sanguíneos para permitir más flujo de oxígeno [7].

\subsection{Utilidad de la remolacha}

La principal utilidad de la remolacha es la obtención de azúcar. Partiendo de su raíz, se obtienen sub productos como pulpa, bagazo, melaza, y cogeneración de electricidad a partir del bagazo, como sub-productos de la extracción del azúcar, con relación al peso fresco de raíz se obtiene aproximadamente: Un 5\% de pulpa, un 4\% de melaza y un 10\% de espumas de cal [8]. Los usos de la remolacha son varias ya sea industrializada o es consumida de la forma ancestral ya sea cocidas o crudas. Se consume en ensaladas o jugos, en la remolacha procesada tenemos en mermeladas, vinos, snacks, vinagre, galletas y la azúcar de remolacha, también es utilizada para el consumo animal, en medicina y ambientales [9].

\subsection{Elaboración del producto}

Al existir una gran relación en el consumo de carbohidratos, en especial de azucares presente en varios tipos de productos que han provocado diferentes tipos de enfermedades se ha buscado una alternativa de consumo de azúcar para las personas con problemas como son galletas de remolacha, en ellas se ha podido encontrar diferentes tipos de aportes nutricional para el cuerpo humano. Las galletas de remolacha demuestran un alto contenido de potasio que es recomendable para aquellas personas que están en constante movimiento físico.

En cuanto a las etiquetas orgánicas predecesoras y los equivalentes nacionales son más reconocibles entre los consumidores. Existe una preocupación creciente por la procedencia de los productos, así como por su forma de elaboración [10]. 


\subsection{Tendencia de consumo y comercialización}

En el año 2012 la industria de las galletas presentó un crecimiento de 5,2\%, en términos monetarios, esto representó USD $\$ 224$ millones y se esperó un crecimiento del 12,7\% en el periodo 2012-2017, lo que representa un crecimiento del 2,54\% anual, el cual se cumplió, se espera que este incremente en 2 puntos porcentuales cada año.

Las importaciones de este tipo de productos ascendieron a USD $\$ 30$ millones, siendo los principales proveedores Colombia (59\%) y Perú (27\%), esto nos da una pauta que la demanda del mercado local no está siendo cubierta por la oferta interna, existiendo posibilidad de mercado por la demanda insatisfecha existente [11].

Analizando el ciclo de vida de la industria, ésta se ubica en etapa de crecimiento, puesto que hoy en día la tendencia de alimentarse es sanamente, además del ingreso de nuevos consumidores con relación a la tasa del crecimiento población en el país, razón para que las empresas invierten en sus plantas de operación, para diversificación de productos.

Observando el consumo de galletas en Ecuador, se determinó que es de 2,5 y 3 kilos de galletas al año, siendo de preferencia consumir galletas de dulce, las mismas que representen el $60 \%$ de facturación y el $40 \%$ de sal. Esas ventas dan como resultado entre 40 y 60 millones en ventas anuales [11].

La comercialización de la remolacha incide en eficientes procesos de industrialización, principalmente se realiza en forma de raíz fresca sin coronas y en forma de cosetas, con volúmenes grandes hasta los industriales y en menos escala para los granjeros, mediante un único canal de distribución es decir del productor al consumidor debido a que la mayoría de la producción es industrializada a nivel interno. Se determina un alto riesgo para emprender un negocio, por diferentes políticas e impuestos existentes [12, 13].

Producto es cualquier bien que se ofrezca a un mercado para su atención, adquisición, uso o consumo y que podría satisfacer un deseo o una necesidad [14]. En ésta investigación el producto a ofrecer son las galletas de remolacha.

El papel de la distribución dentro de la mezcla de marketing consiste en hacer llegar el producto a su mercado meta [15]. La forma de comercializar las galletas de remolacha es a través de varios puntos de venta, como supermercados, considerando que las personas que adquieren este tipo de productos nutritivos, son de ingresos medios Altos.

Las estrategias de marketing a través de las nuevas formas de tecnología, es el apoyo esencial para comercializar el producto.

El marketing digital no sólo ha marcado unas nuevas reglas en el juego, sino que ha ayudado al marketing tradicional a conocer más a fondo a sus clientes [16]. 


\section{Materiales y Métodos}

La presente investigación es de tipo analítico y comparativo, pues se ha obtenido diferentes fuentes de información de la materia prima principal del producto y se ha hecho una comparación nutricional entre tres galletas, por lo cual se llega a definir cuál de los tres productos aporta más beneficios a personas que las consumen.

La elaboración de un documento escrito en el que, de una forma sistemática y estructurada, y previa realización de los correspondientes análisis y estudios, se definen los objetivos a conseguir en un período de tiempo determinado, así como se detallan los programas y medios de acción que son precisos para alcanzar los objetivos enunciados en el plazo previsto. [17]

\subsection{Recolección de la muestra}

Se ha tomado 6 unidades de cada una de las galletas a analizar, se ha estudiado el método de realización y el valor nutricional que puede aportar al consumidor.

El proceso de producción de las galletas de remolacha se basa en simples pasos pues estas galletas están realizadas de manera artesanal remplazando algunos ingredientes que puede beneficiar al cuerpo humano [18].

Se ha utilizado 5 remolachas, las cuales son deshidratadas en el horno para obtener la harina, leche, una pisca de sal, una cucharadita de polvo de hornear, y media cucharada de aceite, el azúcar es opcional pues la remolacha tiene un alto contenido de azúcar que remplaza al azúcar común, se hornea a $150^{\circ} \mathrm{C}$ en 20 min $[19,20]$. 


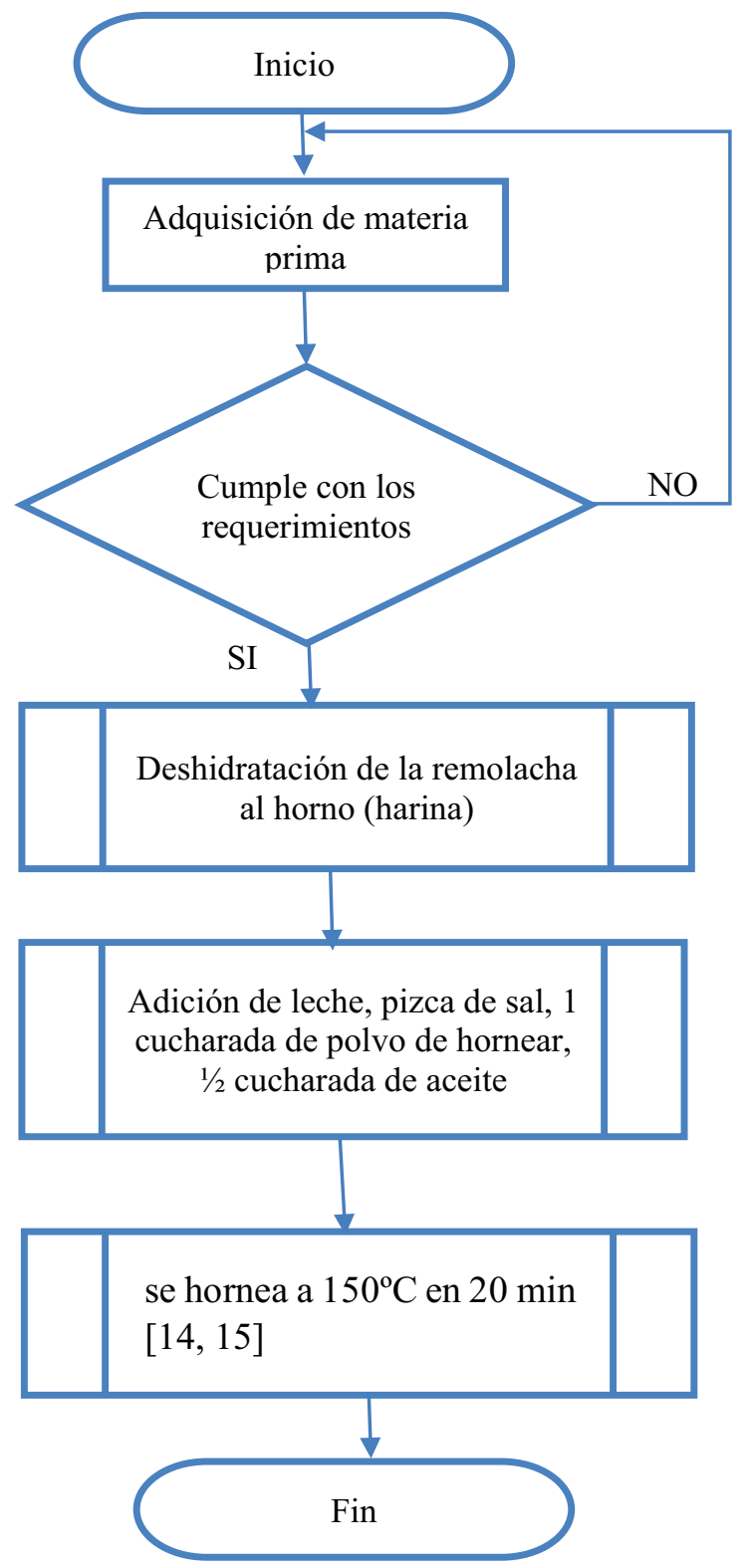

\section{Resultados}

Al concluir la investigación se demuestra que las galletas de remolacha tienen menos hidratos de carbono, en cuanto a las proteínas identificadas las galletas de remolacha tienen menos porcentaje, otro beneficio destacado de la comparación realizada, es que éstas no contienen grasa saturada a diferencia de las galletas rellenitas y las galletas festival con las que se realizó esta comparación, las galletas de remolacha no tienen grasas saturadas; a pesar que las galletas de remolacha no presentan un alto contenido de sodio como con las comparadas, si contiene sodio, potasio, fósforo, calcio además se pudo identificar que las galletas de remolacha no contienen azúcar y también contienen un porcentaje de vitamina B1, mientras que con las comparadas: Galletas rellenitas tienen un mínimo porcentaje, y las galletas festival no poseen esta vitaminas. 


\section{Table 1}

Elaboración de galletas de remolacha para personas veganas [21].

\section{Galleta de remolacha}

\section{Composición}

K calorías

Hidratos de carbono

Fibra

Proteínas

Grasas

Sodio

Calcio

Fosforo

Hierro

Potasio

Vitamina A

Vitamina B1

Vitamina B2

Vitamina B3

Vitamina B12

Vitamina C

\begin{tabular}{ll} 
Cantidad & Porcentaje (\%) \\
$102,12 \mathrm{~g}$ & 50,81 \\
$16,88 \mathrm{~g}$ & 8,39 \\
$1,5 \mathrm{~g}$ & 0,75 \\
$1,78 \mathrm{~g}$ & 0,89 \\
$2,68 \mathrm{~g}$ & 1,33 \\
$81,56 \mathrm{mg}$ & 40,58 \\
$6,44 \mathrm{mg}$ & 3,2 \\
\hline $34,75 \mathrm{mg}$ & 17,29 \\
$0,28 \mathrm{mg}$ & 0,14 \\
$63,3 \mathrm{mg}$ & 31,5 \\
\hline $0 \mathrm{mg}$ & 0 \\
\hline $0,024 \mathrm{mg}$ & 0,01 \\
\hline $0,03 \mathrm{mg}$ & 0,01 \\
\hline $0,51 \mathrm{mg}$ & 2,25 \\
\hline $0 \mathrm{mg}$ & 0 \\
\hline $0,43 \mathrm{mg}$ & 0,21 \\
\hline
\end{tabular}

\section{Table 2}

Información nutricional Rellenitas GN - Galletas [22].

\begin{tabular}{lll} 
Galletas Rellenitas $\mathbf{( 4 2} \mathbf{~ g})$ & Cantidad & Porcentaje (\%) \\
Composición & $31 \mathrm{~g}$ & 73,8 \\
\hline Carbohidratos & $12 \mathrm{~g}$ & 28,57 \\
\hline Azucar & $8 \mathrm{~g}$ & 19,05 \\
\hline Grasas & $4 \mathrm{~g}$ & 9,52 \\
\hline Poliinsaturados & $140 \mathrm{mg}$ & 0,333 \\
\hline Sodio & $4,2 \mathrm{~g}$ & 10 \\
\hline Hierro & $2 \mathrm{~g}$ & 4,76 \\
\hline Proteinas & $210 \mathrm{~g}$ & 50 \\
\hline Calorias & $0,08 \mathrm{mg}$ & 0,00019 \\
\hline Vitamina B1 & $0,11 \mathrm{mg}$ & 0,26 \\
Vitamina B2 & $2,90 \mathrm{mg}$ & 6,9 \\
Vitamina B3 & $9 \mathrm{ug}$ & $9,523 \mathrm{E}-06$ \\
Vitamina B9 & $1,10 \mathrm{mg}$ & 0,0026 \\
\hline Vitamina E & $4 \mathrm{ug}$ & 0,0000095 \\
Vitamina K & $42 \mathrm{mg}$ & 0,1 \\
\hline Magnesio & $66 \mathrm{mg}$ & 0,16 \\
\hline Calcio & &
\end{tabular}


Table 3

Informe de práctica para la obtención del título de ingeniería en alimentos [23].

\begin{tabular}{llll} 
Galletas festival $\mathbf{( 4 2} \mathbf{~ g )}$ & Cantidad & Porcentaje (\%) \\
Composición & $36 \mathrm{~g}$ & 17,91 \\
\hline Carbohidratos & $0 \mathrm{~g}$ & 0 \\
\hline Fibra dietética & $17 \mathrm{~g}$ & 8,46 \\
\hline Azúcar & $8 \mathrm{~g}$ & 3,98 \\
\hline Grasas & $4 \mathrm{~g}$ & 1,99 \\
\hline Saturadas & $0 \mathrm{~g}$ & 0 \\
\hline Polinsaturados & $3 \mathrm{~g}$ & 1,49 \\
\hline Mono saturados & $0 \mathrm{~g}$ & 0 \\
\hline Trans & $2 \mathrm{~g}$ & 0,99 \\
\hline Proteínas & $110 \mathrm{mg}$ & 54,73 \\
\hline Sodio & $0 \mathrm{mg}$ & 0 \\
\hline Potasio & $0 \mathrm{mg}$ & 0 \\
\hline Colesterol & $0 \%$ & 0 \\
Vitamina A & $0 \%$ & 0 \\
Vitamina C & $0 \%$ & 0 \\
Calcio & $0 \%$ & 0 \\
Hierro & & \\
& 0 & 0 & 0 \\
\hline
\end{tabular}

\section{Discusión}

Según el análisis de valor nutritivo investigado entre las galletas de remolacha y las reconocidas galletas festival, se pudo determinar que las galletas de remolacha tienen $10 \%$ más proteínas y $15 \%$ más que las galletas festival. Las galletas festival contienen mayor porcentaje de hidratos de carbono que las galletas de remolacha con un 9,52\%, concordando con investigaciones realizadas que apoyan la teoría [24], en cuanto en grasas las galletas festival contienen un porcentaje del 3,98\% que también incluyen las grasas saturadas de 1,99\% y de grasas monosaturados con 1,49\%, mientras que las galletas de remolacha presentan el 1,33\% de grasa, en sodio las galletas de remolacha presentan un porcentaje del $14,15 \%$ menos que la galleta festival, las galletas festival también cuentan con un porcentaje del $8,46 \%$ de azúcar mientras que las galletas de remolacha no contienen azúcar, las galletas de remolacha contienen algunos otros aportes nutricionales que entre ellas tenemos Calcio 3,20\%, fosforo $17,29 \%$, hierro 0,14\%, potasio $31,5 \%$, vitamina B1 0,01\%, vitamina B2 0,01\%, vitamina B3 con el 2,25\% y la vitamina $\mathrm{C}$ con el $0,21 \%$.

Estos resultados permiten afirmar que las galletas de remolacha tienen un inferior margen de aporte de carbohidratos, pero un alto valor nutricional, coincidiendo con varias investigaciones secundarias [25]. 


\section{Table 4}

Modelo canvas: Comercialización galletas de remolacha.

\begin{tabular}{|c|c|}
\hline Socios clave & Actividades clave \\
\hline \multirow[t]{3}{*}{$\begin{array}{l}\text { Proveedores de } \\
\text { verduras. } \\
\text { Proveedores de } \\
\text { leche. } \\
\text { Proveedores de } \\
\text { huevos. Entre } \\
\text { otros. }\end{array}$} & $\begin{array}{l}\text { Campañas } \\
\text { publicitarias. } \\
\text { Degustaciones en } \\
\text { puntos de venta. } \\
\text { Promociones por } \\
\text { lanzamiento. }\end{array}$ \\
\hline & Recursos clave \\
\hline & $\begin{array}{l}\text { Materia prima } \\
\text { Harina } \\
\text { Mantequilla } \\
\text { Canela Levadura } \\
\text { Huevos Verduras } \\
\text { Sal Leche Polvo } \\
\text { de hornear Aceite } \\
\text { Maquinaria } \\
\text { Batidora Horno } \\
\text { Procesador de } \\
\text { comida }\end{array}$ \\
\hline
\end{tabular}

Estructura de costos

Los principales costos, recaen en la compra de materia prima y mano de obra para la fabricación del producto.

Propuesta de valor
Ofrecer al cliente un
snack saludable con
alto contenido de
nutrientes, que
además de
satisfacer el hambre,
aporte energía y
vitaminas por los
ingredientes
utilizados.

\section{Relaciones con los} clientes

Contacto

permanente con los

posibles clientes,

mediante campañas

publicitarias, que

proporcionen la

información

necesaria sobre el

producto.

\section{Canales}

Se podría utilizar

canales de

distribución

indirectos. Ejemplo:

Supermercados.

\section{Conclusiones}

1. A través del análisis comparativo, se identifica que las galletas de remolacha, son un alimento que poseen una gran cantidad de nutrientes, aportando dosis significativas de vitaminas, minerales y antioxidantes, para las personas que lo consuman, teniendo en cuenta el estilo de vida saludable como tendencia actual.

2. Los competidores ofrecen productos con características saludables a base de elementos integrales en algunos casos, sin embargo, hay que priorizar las vitaminas que ofrecen los vegetales, por lo que se constituye en la principal ventaja competitiva de éste estudio analítico.

3. Se examinó la industria de galletas, la cual ha mantenido una tendencia creciente en el mercado ecuatoriano, con resultados económicos favorables para la industria, razón por la cual se convierte en un motivo para emprender en ésta área.

4. La comercialización de galletas de remolacha, basados en el interés de las personas que hoy en día tratan de mantener buenos hábitos alimenticios, tendrán una buena aceptación de consumo, apoyados principalmente en la elaboración de un estudio de mercado, y un modelo de negocio. 


\section{References}

[1] Cabezas et al. Azúcares adicionados a los alimentos: Efectos en la salud y regulación mundia. Revisión de la literatura. 2015.

[2] Arias, Rodrigues D. Principales factores de riesgo de diabetes mellitus tipo II en adultos mayores ingresado en el servicio de medicina interna del hospital provincial general de docente de Riobamba periodo enero 2009-diciembre 2011. Riobamba; 2013.

[3] Tiradritti F, Luca A. 2006. La historia del museo egipcio. en tesoros de egipto. Libsa; 2006.

[4] Tubon J. Evaluación del potencial nutritivo de mermelada elaborado a base de remolacha. Riobamba; 2011.

[5] Urbano L. Elaboración de snack nutraceútica de quinua con remolacha como colorante. Riobamba; 2014.

[6] Borrego JVM. Horticultura herbácea especial. $5^{\text {th }}$ edición. España: Editorial Mundi- Prensa; 2002.

[7] Guffantte M. Introducción de la remolacha en la elaboración de cocteles y postres de la aceptabilidad en la escuela de gastronomía, 2012. Riobamba; 2013.

[8] Wylie LJ, Kelly J, Bailey SJ et al. Beetroot juice and exercise: [harmacodynamic and dose response relationships. J Appl Physiol. 2013;115(3):325-336

[9] Una H, et al. Dietético nitrato aumenta tetánico contracción rápida músculo. J Physiol. 2012.

[10] Romero I, Díaz V, Aguirre A. Fortalecimiento de la cadena de valor de los snacks nutritivos con base en fruta deshidratada en el Salvador. 2016. Recuperado de: https://repositorio.cepal.org/bitstream/handle/ 11362/40251/1/S1600668_es.pdf

[11] OCDE/FAO. Perspectivas Agrícolas 2013-2022. Texcoco: Universidad Autónoma Chapingo; 2013.

[12] Importaciones de galletas a Ecuador. ProEcuador. 2015 October 10. Retrieved from: www.proecuador. gob.ec/sector 1-6

[13] Clasificación de industrias. El Universo. 2015 October 12. Available from: http://www.eluniverso.com/ 2007/08/20/0001/9DCF43089F3D14A90B992dF41EF7B988.html)

[14] Kotler P, Armstrong G. Marketing. México: Pearson; 2012.

[15] Stanton. Fundamentos de marketing. México: Mc Graw-Hill Interamericana; 2007.

[16] Cangas. Marketing digital: Tendencias en su apoyo al e-commerce y sugerencias de implementación. Santiago. Chile: Universidad de Chile; 2010.

[17] Muniz GR. Marketing en el Siglo XXI. 5th edition. España: Centro de Estudios Financieros; 2014.

[18] El Comercio. Factibilidad de negocios. 2015 October 7. Available from: http://www.elcomercio.com/ actualidad/ecuador-ranking-facilidades-negocios-estudio.html

[19] Chicaiza J. Proyecto de factibilidad para la implementación de una cafetería con enfoque repotencializador utilizando productos autóctonos de la región sierra, en el cantón Quito, provincia de Pichincha 2017. 2017. Retrieved from: http://dspace.espoch.edu.ec/handle/123456789/11534

[20] Preparar galletas de remolacha y gotas de chocolate. Pitoquito. 2017.

[21] Paez. Elaboración de galletas de remolacha para personas veganas. 2017.

[22] Información nutricional Rellenitas GN- Galletas. Fuente: Under Amour; 2019.

[23] Venavidez. Informe de práctica para la obtención del título de ingeniería de alimentos. 2012.

[24] Rnakin J, D. Glycemic index and metabolism. Volumen 10 SSE 64. 1997.

[25] Miller J. Diets with a low glycemic index: Theory to practice. 